\title{
POTENCIAL DE REDUÇÃO DE EMISSÃO DE EQUIVALENTE DE CARBONO DE UMA UNIDADE SUINÍCOLA COM BIODIGESTOR ${ }^{1}$
}

\author{
ANDRÉ R. ANGONESE ${ }^{2}$, ALESSANDRO T. CAMPOS ${ }^{3}$, ROSILENE A. WELTER ${ }^{4}$
}

RESUMO: O objetivo do presente trabalho foi avaliar o potencial de reduções de emissão de carbono, em $\mathrm{tCO}_{2} \mathrm{eq} \mathrm{ano}^{-1}$, e estimar o valor financeiro obtido anualmente para o total de suínos da granja e o valor médio anual por animal em suinocultura, em sistema de recria e engorda, com capacidade instalada para 600 animais. O sistema de tratamento dos dejetos é realizado de forma integrada, possibilitando a reciclagem dos nutrientes, em que o biodigestor anaeróbio tubular estabiliza a matéria orgânica, produzindo metano e biofertilizante no processo. A estimativa foi efetuada baseando-se na metodologia AM0006, aprovada pelo Conselho Executivo do Mecanismo de Desenvolvimento Limpo do IPCC. O potencial de redução de emissão de $\mathrm{CO}_{2}$ equivalente por ano foi de $325,16 \mathrm{t}$, correspondendo à redução de emissão de $0,54 \mathrm{tCO}_{2}$ eq animal ${ }^{-1}$ ano $^{-1}$, e a possibilidade de ganho financeiro com a comercialização desses créditos de carbono de aproximadamente $\mathrm{R} \$ 5,31$ animal $^{-1} \mathrm{ano}^{-1}$.

PALAVRAS-CHAVE: Protocolo de Kioto, dejetos de suínos, biodigestor.

\section{GREENHOUSE GAS EMISSION MITIGATION FROM A PIG FARM WITH DIGESTOR}

ABSTRACT: Greenhouse gas emissions ( $\mathrm{tCO} 2$ eq $_{\text {year }}{ }^{-1}$ ) and the money earned annually with the total amount of pigs and the average per pig were evaluate. The pig farm has about 600 animals. The waste treatment sistem is integrated making possible the nutrients recycling. The tubular anaerobic digestor degrade organic matter producing methane and fertilizer. The estimation made was based on the methodology AM0006 approved by Clean Development Mechanism Executive Board. The mitigation potential calculated was $325.16 \mathrm{tCO}^{2}$ eq year ${ }^{-1}$, or $0.54 \mathrm{tCO}^{2}$ eq year ${ }^{-1}$. The financial income was approximately $\mathrm{R} \$ 5.31$ animal $^{-1}$ year $^{-1}$.

KEYWORDS: Kyoto Protocol, pig waste, digestor.

\section{INTRODUÇÃO}

As atividades do homem estão acentuando as concentrações dos gases atmosféricos responsáveis pelo efeito estufa, ocasionando aumento da temperatura da superfície terrestre. Devido à necessidade de se desenvolverem políticas e instrumentos legais internacionais sobre a questão do clima, foi adotada a Convenção-Quadro das Nações Unidas sobre Mudanças do Clima (MIGUEZ, 2001). São passíveis de negociação emissões dos gases listados no Anexo A do Protocolo de Kioto: Dióxido de Carbono $\left(\mathrm{CO}_{2}\right)$, Metano $\left(\mathrm{CH}_{4}\right)$, Óxido Nitroso $\left(\mathrm{N}_{2} \mathrm{O}\right)$, Hidrofluorcarbonos (HFCs), Perfluorcarbonos (PFCs) e Hexafluoreto de Enxofre $\left(\mathrm{SF}_{6}\right.$ ), sendo referenciados em $\mathrm{CO}_{2}$ equivalente, conforme estabelecido pelo Painel Intergovernamental sobre mudanças do clima (Intergovernmental Panel on Climate Change - IPCC). A agricultura e a pecuária contribuem, predominantemente, para as emissões antrópicas de metano $\left(\mathrm{CH}_{4}\right)$, monóxido de carbono $(\mathrm{CO})$, óxido nitroso e óxidos de nitrogênio $\left(\mathrm{NO}_{\mathrm{x}}\right)$ à atmosfera terrestre (LIMA et al., 2001).

\footnotetext{
${ }^{1}$ Extraído da Dissertação de Mestrado do primeiro autor.

${ }^{2}$ Engo Florestal, Mestrando, GPEA/CCA/UNIOESTE, Largo São Vicente de Paulo, 1056/72, Toledo - PR, Fone: (0XX45) 3252.2551, aangonese@yahoo.com.br

${ }^{3}$ Eng $^{\text {o }}$ Agrícola, Prof. Adjunto, Departamento de Agronomia, DAG/UFVJM, Diamantina - MG, atcampos3@yahoo.com.br

${ }^{4}$ Acadêmica de Eng ${ }^{a}$ Química, UNIOESTE, leneaw@ yahoo.com.br

Recebido pelo Conselho Editorial em: 1-12-2005

Aprovado pelo Conselho Editorial em: 22-8-2007
} 
A degradação biológica do material orgânico (fezes, urina, ração e outros) produz gases tóxicos que podem afetar a saúde, o desempenho dos suínos e a qualidade do ar. O dióxido de carbono, o metano, o óxido nitroso e a amônia são os gases de maior interesse para a suinocultura atualmente. $\mathrm{O}$ dióxido de carbono é mais pesado do que o ar, inodoro e asfixiante. A concentração máxima admissível nas edificações é de 3.500 ppm (BENEDI, 1986). O metano é um gás inodoro, mais leve que o ar, asfixiante, inflamável e explosivo, requerendo extremo cuidado no seu manejo. Concentrações acima de 500 mil ppm provocam dores de cabeça em humanos (USDA, 1994, citado por PERDOMO et al., 2001). O potencial de aquecimento global aprovado e com referência ao dióxido de carbono é de 21 vezes maior (IPCC, 1997). Óxido nitroso é um gás emitido durante o tratamento dos dejetos de suínos líquidos, com grande influência nas alterações climáticas, especialmente na destruição da camada de ozônio. A emissão de óxido nitroso referente à produção de suínos representa parcela significativa da emissão total atribuída à agricultura nos grandes centros produtores. $\mathrm{O}$ potencial de aquecimento global é de 310 vezes o potencial de aquecimento do $\mathrm{CO}_{2}$ (IPCC, 1997).

A estrutura atual dos sistemas produtivos de suínos, com a concentração de animais em pequenas áreas, gerando grandes excedentes de dejetos, demanda áreas relativamente grandes para o seu reaproveitamento agronômico (OLIVEIRA, 2004). Sendo assim, a utilização de biodigestores no meio rural tem merecido destaque devido aos aspectos de saneamento e geração de energia, além de estimular a reciclagem orgânica e de nutrientes (LUCAS JÚNIOR, 1994). Atualmente, os sistemas de armazenamento e tratamento de resíduos de suínos existentes no Sul do Brasil consistem de lagoas (anaeróbias, facultativas e aeróbias), fossas internas, bioesterqueiras e esterqueiras. As esterqueiras com expressiva predominância, geralmente mal dimensionadas, ou sem nenhum projeto, não levam em consideração o volume de resíduos produzidos e o tempo necessário para a estabilização desse (LIMA et al., 2002). O custo médio de implantação era de US\$ 50,00 $\mathrm{m}^{-3}$ para biodigestor e US\$ $5,00 \mathrm{~m}^{-3}$ para lagoa anaeróbia-padrão, conforme PERDOMO et al. (2003).

Algumas das vantagens da digestão anaeróbia são: alta redução de demanda bioquímica de oxigênio (DBO), produção de biofertilizante, pequena produção de lodo, baixos custos operacionais e de investimento, e possibilidade de sistemas descentralizados de tratamento de resíduos (OLIVEIRA, 2004).

ERNST et al. (1990) observaram que a produção de metano, por digestão anaeróbia, em biodigestores de alimentação contínua, solucionou problemas de odores e geração de energia alternativa em Iowa, nos Estados Unidos da América.

A Agência de Proteção Ambiental Americana (USEPA, 1994) estimou que cerca de 14\% da emissão global de gás metano tem origem em atividades relacionadas à produção animal. Além do metano, os gases de nitrogênio também apresentam alto potencial de aumento do efeito estufa. Esses gases são produzidos durante a estabilização dos dejetos. Os microorganismos utilizam o nitrato como aceptor de elétrons em sua cadeia respiratória, transformando-o em formas gasosas de $\mathrm{N}$, como o óxido nitroso e $\mathrm{N}_{2}$ (OLIVEIRA et al., 2004). Dessa forma, os sistemas de produção de animais (notadamente os confinados) representam crescente fonte de emissão de $\mathrm{N}_{2} \mathrm{O}$ na agricultura (OENEMA et al., 2005).

O objetivo do presente trabalho foi avaliar o potencial de reduções de emissão de carbono, em $\mathrm{tCO}_{2}$ eq ano $^{-1}$, e estimar o valor econômico obtido anualmente, em unidade de terminação de suínos, utilizando biodigestores para o tratamento dos dejetos.

\section{MATERIAL E MÉTODOS}

O trabalho foi desenvolvido na Fazenda Vale dos Ipês, município de Ouro Verde do Oeste, à

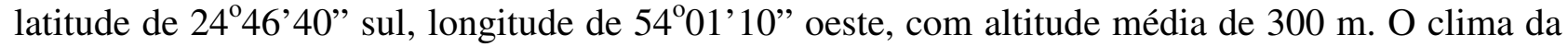
região é do tipo Cfa (classificação climática de Köeppen), subtropical, úmido, mesotérmico, com precipitação média anual de 1.600 a 1.700 mm (IAPAR, 1994). 
Foi analisada uma unidade de terminação de suínos (UT), com recria e engorda de 600 animais, em confinamento total, conduzido em instalação aberta lateralmente, com cobertura de telhas cerâmicas, suportada por estruturas de madeira, com o tratamento dos resíduos realizado por meio de biodigestor.

A condução dos dejetos ao biodigestor é realizada de forma contínua, por meio de tubos de policloreto de vinila (PVC) de $150 \mathrm{~mm}$, o que evita a entrada da água da chuva e do pátio. Todo o sistema funciona por gravidade, não necessitando de bombeamento para o funcionamento. $\mathrm{O}$ biogás é coletado por meio de mangueira de polietileno de $50 \mathrm{~mm}$ e armazenado em bolsas de PVC.

O biodigestor é formado por três tanques cilíndricos, sendo um de $20 \mathrm{~m}^{3}$, fabricado em fibra de vidro, e dois "tipo tanque" (usados em postos de combustível) de $15 \mathrm{~m}^{3}$ cada, construídos em aço carbono, com entrada e saída para fluxo contínuo dos resíduos, sendo o diâmetro da tubulação de $200 \mathrm{~mm}$, provido de tubulação na parte superior para a coleta do biogás, sendo o efluente do biodigestor depositado em um tanque para armazenagem.

\section{Metodologia de cálculo utilizada}

A metodologia para a estimativa da redução de emissão de carbono, em $\mathrm{tCO}_{2} \mathrm{eq} \mathrm{ano}{ }^{-1}$, foi fundamentada na linha de base para sistemas de manejo de dejetos, referendado como AM0006 "Redução de emissão de gases de efeito estufa para sistemas de manejo de dejetos" (UFCCC, 2004), dentro do Mecanismo de Desenvolvimento Limpo (MDL), instituído pelo Protocolo de Kioto.

Seguindo os conceitos descritos na metodologia, as emissões consideradas nessa análise incluem a emissão de metano de lagoas anaeróbias abertas, fugas de $\mathrm{CH}_{4}$ devido às perdas no biodigestor e emissões de $\mathrm{N}_{2} \mathrm{O}$ pela linha de base e pelos cenários do projeto proposto (Figuras $1 \mathrm{e}$ 2). $\mathrm{O} \mathrm{CO}_{2}$ das fugas e perdas, gerado a partir da digestão anaeróbia, não representa qualquer diferença em volumes de emissão entre os dois cenários, pois não existem possíveis transformações adicionais pela queima desse componente. Na Figura 1, são demonstrados os limites para o cenário da linha de base e, na Figura 2, para o projeto proposto.

A fronteira do projeto inclui somente as emissões do sistema de manejo dos dejetos, considerando os dejetos de suínos produzidos nas instalações e encaminhados dentro do sistema de tratamento. A emissão de carbono, originada da queima do metano, é considerada na avaliação (Figura 2). Essa aceitação é baseada no fato de que a alimentação dos animais tem fonte renovável de fornecimento e não-fóssil. O potencial de fugas das emissões relatadas para o sistema de digestão anaeróbia e o encanamento foram incluídos dentro da fronteira do sistema. A aplicação do biofertilizante no solo é feita em área de pastagem próxima, mas fora do limite do sistema.

A rede de emissões é diferente entre o sistema de tratamento de dejetos da linha de base e do projeto proposto.

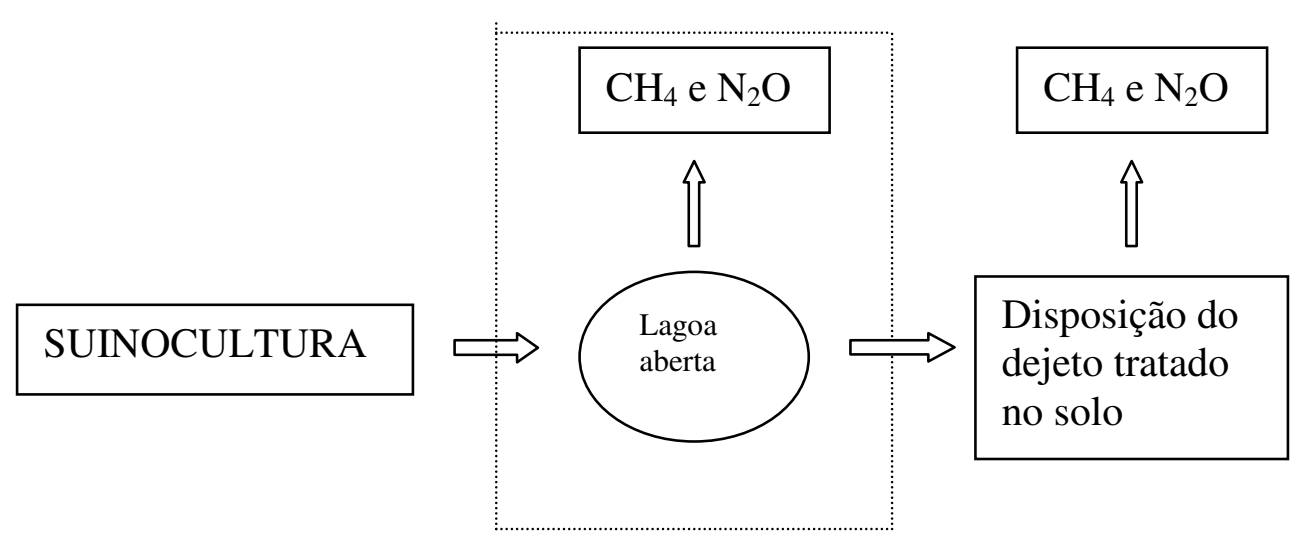

FIGURA 1. Esquema dos limites de um cenário com lagoa tradicional (linha de base). 


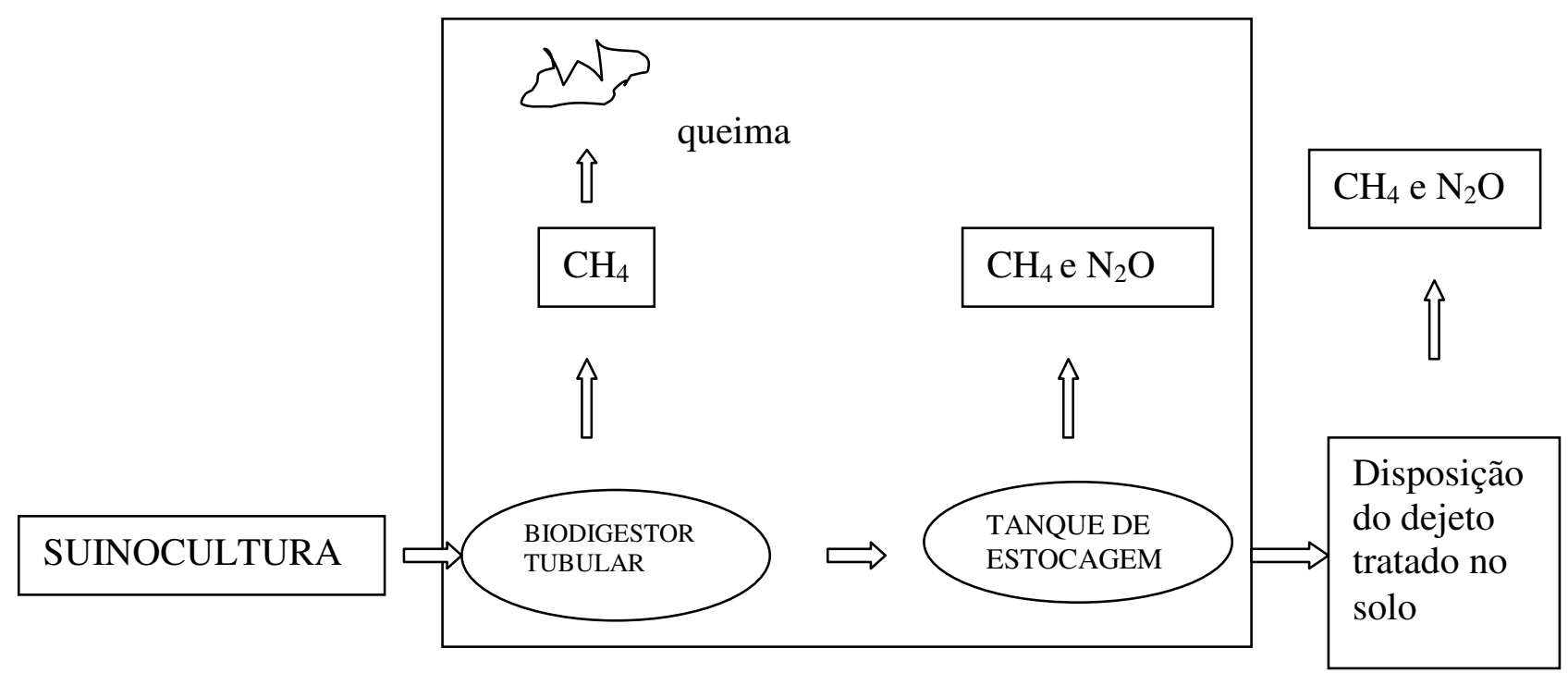

FIGURA 2. Esquema dos limites de fronteira do sistema proposto.

\section{Equações usadas para estimar as emissões do projeto}

Para o tratamento de dejetos no biodigestor, as emissões foram calculadas conforme abordagem da eq.(1). Alternativamente, para o cálculo da emissão de metano para o tanque de estocagem, ou subseqüentes do sistema de tratamento, é utilizada a eq.(2).

As emissões de $\mathrm{CH}_{4}$ relacionadas ao biodigestor anaeróbio são estimadas a partir da eq.(1):

$\mathrm{E}_{\mathrm{CH} 4, \mathrm{~mm}, 1, \mathrm{y}}=\mathrm{SV} \mathrm{B}_{\mathrm{o}} \mathrm{D}_{\mathrm{CH} 4} \mathrm{FCM}_{1} \mathrm{PAG}_{\mathrm{CH} 4} \mathrm{~N}_{\mathrm{y}} 3651000^{-1}$

As emissões de $\mathrm{CH}_{4}$ relacionadas à lagoa de armazenamento são estimadas a partir da eq.(2):

$\mathrm{E}_{\mathrm{CH} 4, \mathrm{~mm}, 2, \mathrm{y}}=\mathrm{SV}[1-\mathrm{Rsv}] \mathrm{B}_{\mathrm{o}} \mathrm{D}_{\mathrm{CH} 4} \mathrm{FCM}_{2} \mathrm{PAG}_{\mathrm{CH} 4} \mathrm{~N}_{\mathrm{y}} 3651000^{-1}$ em que,

$\mathrm{E}_{\mathrm{CH} 4, \mathrm{~mm}, 1, \mathrm{y}}$ - emissão de $\mathrm{CH}_{4}$, relacionada ao biodigestor anaeróbio, durante o ano y, toneladas de $\mathrm{CO}_{2}$ equivalente $\left(\mathrm{tCO}_{2} \mathrm{eq}\right)$;

$\mathrm{E}_{\mathrm{CH} 4, \mathrm{~mm}, 2, \mathrm{y}}$ - emissão de $\mathrm{CH}_{4}$, relacionada à lagoa de armazenamento, durante o ano y, toneladas de $\mathrm{CO}_{2}$ equivalente $\left(\mathrm{tCO}_{2} \mathrm{eq}\right)$;

$\mathrm{PAG}_{\mathrm{CH} 4}$ - potencial aquecimento global aprovado de $\mathrm{CH}_{4}$;

$\mathrm{FCM}_{1}$ - fator de conversão do metano para tratamento de dejetos em biodigestor anaeróbio;

$\mathrm{FCM}_{2}$ - fator de conversão do metano para tratamento de dejetos em lagoa de armazenamento;

$\mathrm{D}_{\mathrm{CH} 4}$ - densidade do metano $\left(0,67 \mathrm{~kg} \mathrm{~m}^{-3}\right)$ na temperatura de $20{ }^{\circ} \mathrm{C}$ e a 1 atm de pressão;

$\mathrm{SV}$ - sólidos voláteis excretados por dia $\left(\mathrm{kg}_{\text {animal }}{ }^{-1} \mathrm{dia}^{-1}\right)$ para o ano;

$\mathrm{R}_{\mathrm{SV}}$ - redução relativa de sólidos voláteis na segunda lagoa de estocagem, percentual;

$\mathrm{B}_{\mathrm{o}}$ - capacidade de produção máxima para o dejeto por animal para uma população de animais definida, $\mathrm{m}^{3}$ de $\mathrm{CH}_{4} \mathrm{~kg}^{-1}$, e

$\mathrm{N}_{\mathrm{y}}$ - população de animais definida por ano. eq.(3):

As emissões de $\mathrm{N}_{2} \mathrm{O}$ da lagoa anaeróbia e de fugas no armazenamento são estimadas pela

$\mathrm{E}_{\mathrm{N} 2 \mathrm{O}, \mathrm{mm}, 1, \mathrm{y}}=\mathrm{PAG}_{\mathrm{N} 2 \mathrm{O}} \mathrm{N}_{\mathrm{EXy}} \mathrm{N}_{\mathrm{y}} \mathrm{FE}_{\mathrm{N} 2 \mathrm{O}, \mathrm{mmmi}} \mathrm{FC}_{\mathrm{N} 2 \mathrm{O}-\mathrm{N}, \mathrm{N}} 1000^{-1}$

em que, 
$\mathrm{E}_{\mathrm{N} 2 \mathrm{O}, \mathrm{mm}, 1, \mathrm{y}}$ - emissões de óxido nitroso para o estágio de lagoa anaeróbia do sistema de manejo de dejetos, toneladas de $\mathrm{CO}_{2}$ equivalentes por ano;

$\mathrm{PAG}_{\mathrm{N} 2 \mathrm{O}}$ - potencial de aquecimento global aprovado para $\mathrm{N}_{2} \mathrm{O}$;

$\mathrm{FE}_{\mathrm{N} 2 \mathrm{O}, \mathrm{mmmi}}$ - fator de emissões para o primeiro estágio do tratamento do sistema de manejo de dejetos, $\mathrm{kg}$ de $\mathrm{N}_{2} \mathrm{O}-\mathrm{N} \mathrm{kg} \mathrm{N}{ }^{-1}$;

$\mathrm{N}_{\mathrm{EXy}}$ - média anual de excreção de nitrogênio por animal para uma população definida em $\mathrm{kg}$ de $\mathrm{N}_{\text {animal }}{ }^{-1}$ ano $^{-1}$, para o ano y;

$\mathrm{FC}_{\mathrm{N} 2 \mathrm{O}-\mathrm{N}, \mathrm{N}}$ - fator de conversão de $\mathrm{N}_{2} \mathrm{O}-\mathrm{N}$ para $\mathrm{N}(44 / 28)$, e

$\mathrm{N}_{\mathrm{y}}$ - população de animais definida para o ano y.

O conteúdo de sólidos voláteis nos dejetos não-tratados é dado pela eq.(4):

$\mathrm{SV}_{\text {local }}=\left(\mathrm{W}_{\text {local }} / \mathrm{W}_{\text {padrão }}\right) \mathrm{SV}_{\text {padrão }}$

em que,

$\mathrm{SV}_{\text {local }}$ - excreção ajustada de sólidos voláteis por dia, em base de matéria seca, para um rebanho de população definida, no local do projeto, $\mathrm{kg}_{\text {animal }}{ }^{-1} \mathrm{dia}^{-1}$;

$\mathrm{W}_{\text {local }}$ - peso médio do animal local para uma população definida, $\mathrm{kg}$;

$\mathrm{W}_{\text {padrão }}$ - valor-padrão do peso médio do animal para uma população definida $\mathrm{kg}$, e

$\mathrm{SV}_{\text {padrão }}$ - valor-padrão (IPCC) para excreção de sólidos voláteis por dia, em base de matéria seca, para um rebanho de população definida, $\mathrm{kg}_{\text {animal }}{ }^{-1} \mathrm{dia}^{-1}$.

A taxa de excreção de nitrogênio para dejetos não-tratados, em $\mathrm{kg}_{\text {cabeça }}{ }^{-1}$ dia $^{-1}$, é dada pela eq.(5):

$\mathrm{N}_{\text {EXlocal }}=\left(\mathrm{W}_{\text {local }} / \mathrm{W}_{\text {padrão }}\right) \mathrm{N}_{\text {EXpadrão }}$

em que,

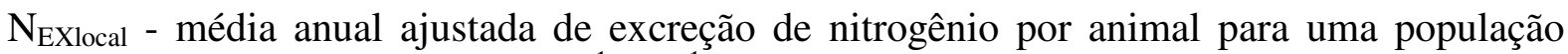
definida, $\mathrm{kg}$ de $\mathrm{N}$ animal ${ }^{-1}$ ano $^{-1}$;

$\mathrm{W}_{\text {local }}$ - peso médio do animal local para uma população definida, $\mathrm{kg}$;

$\mathrm{W}_{\text {padrão }}$ - valor-padrão do peso médio do animal para uma população definida $\mathrm{kg}$, e

$\mathrm{N}_{\text {EXlocal }}$ - valor-padrão (IPCC) para a excreção de nitrogênio por animal para uma população definida, $\mathrm{kg}$ de $\mathrm{N}$ animal ${ }^{-1}$ ano $^{-1}$.

A metodologia adotada envolve incertezas na estimativa das emissões, como alguns parâmetros-chave, que são freqüentemente dúbios e, muitas vezes, apenas valores-padrão são acessíveis. Por essa razão, foram escolhidos de modo a evitar superestimações e outros foram definidos conforme as práticas locais e o contexto do projeto.

\section{RESULTADOS E DISCUSSÃO}

\section{Valores das variáveis}

\section{Sólidos Voláteis (SV)}

De acordo com a energia e a alimentação média obtida na granja, o valor-base usado para os sólidos voláteis de dejetos não-tratados é a taxa do IPCC (1997) para países desenvolvidos. Essa suposição alvitra que as práticas usadas na maioria das granjas na região oeste do Paraná, incluindo o tipo de alimentação, a taxa e a herança genética, são similares às práticas dos países desenvolvidos. A taxa de sólidos voláteis produzidos, calculada para um peso representativo de $82 \mathrm{~kg}$ animal ${ }^{-1}$, foi ajustada para a média de peso dos suínos da granja em estudo, sendo de $57,4 \mathrm{~kg}$ animal $^{-1}$. 
$\mathbf{B}_{\mathbf{0}}$

O valor-padrão $B_{0}$, que representa o máximo de capacidade de produção de metano a partir dos dejetos por animal, foi obtido em IPCC (1997). Considerando as práticas adotadas na granja, optou-se por empregar o valor representativo para países desenvolvidos $\left(0,45 \mathrm{~m}^{3} \mathrm{~kg}^{-1}\right)$.

\section{Fator de Conversão de Metano (FCM)}

Para o FCM, foi usado o valor-padrão do IPCC (1997) de 0,9\% para clima temperado, específico para a região, conforme IBGE (2005).

\section{Taxa de excreção de nitrogênio $\left(\mathrm{N}_{\mathrm{Ex}}\right)$}

A taxa de excreção do nitrogênio foi obtida no IPCC (1997). A taxa utilizada, de $20 \mathrm{~kg}$ animal $^{-1} \mathrm{dia}^{-1}$, para países desenvolvidos, foi corrigida para a média de peso dos suínos da granja.

\section{Valor-padrão do Fator de Emissão para o $\mathbf{N}_{2} \mathrm{O}$ (FE)}

$\mathrm{O}$ valor-padrão do parâmetro usado para FE $3\left(0,001 \mathrm{~kg} \mathrm{de}_{2} \mathrm{O}-\mathrm{N} \mathrm{kg}^{-1}\right)$ foi encontrado no IPCC (1997).

\section{Cálculo das emissões de gases de efeito estufa por fontes}

As emissões de gases de efeito estufa, incluídas na fronteira do sistema, foram calculadas separadamente para o projeto e para a linha de base do sistema de tratamento de dejetos, usando a mesma metodologia aprovada. A redução de emissões resulta da diferença entre as emissões do projeto e da linha de base.

$\mathrm{O}$ fator que mais afetou as emissões de metano, para manejo de dejetos de suínos, foi a quantidade de dejetos produzidos e a porção de dejetos que pode decompor-se em condições anaeróbias. O tipo de sistema de tratamento de dejetos usado e o clima, principalmente a temperatura, são os fatores que determinam a extensão da decomposição anaeróbia que é adotada para o local.

$\mathrm{O}$ óxido nitroso $\left(\mathrm{N}_{2} \mathrm{O}\right)$ do manejo de dejetos é produzido da combinação do processo de nitrificação e denitrificação que ocorre no nitrogênio dos dejetos. A maioria do nitrogênio do dejeto está na forma de amônia $\left(\mathrm{NH}_{3}\right)$. A nitrificação ocorre aerobicamente e converte essa amônia em nitrato, enquanto a denitrificação ocorre anaerobiamente, e converte o nitrato em óxido nitroso. A temperatura, o pH, a demanda bioquímica de oxigênio (DBO) e a concentração de nitrogênio afetam a taxa de geração de óxido nitroso. A amônia pode ser emitida dentro das instalações dos animais, nas instalações de tratamento e no armazenamento dos dejetos e na aplicação no solo (KIRCHMANN et al., 1998).

Melhorar eficientemente o uso do nitrogênio, combinado com a digestão anaeróbia dos dejetos de animais para geração de biogás, são as ações mais viáveis para a mitigação do efeito do óxido nitroso (OENEMA et al., 2005).

Para representar as emissões de cada estágio do tratamento nos cenários do projeto e da linha de base, procederam-se os cálculos conforme equações, variáveis e valores apresentados anteriormente, chegando-se aos seguintes resultados.

TABELA 1. Emissões em $\mathrm{tCO}_{2} \mathrm{eq} \mathrm{ano}^{-1}$ detalhadas do projeto, por fontes.

\begin{tabular}{lc}
\hline \multicolumn{1}{c}{ Emissões do Projeto } & $\mathrm{tCO}_{2} \mathrm{eq}_{\text {ano }}{ }^{-1}$ \\
\hline Emissões e perdas do biodigestor de $\mathrm{CH}_{4}$ & 24,26 \\
$\mathrm{CH}_{4}$ da lagoa secundária & 87,35 \\
$\mathrm{~N}_{2} \mathrm{O}$ da lagoa secundária & 4,31 \\
\hline Emissões Totais do Projeto & 115,92 \\
\hline
\end{tabular}


Para o cenário do projeto utilizando o biodigestor como tratamento primário e uma lagoa secundária para depósito, determinaram-se os valores estimativos potenciais de fugas e perdas de metano no biodigestor em $24,26 \mathrm{tCO}_{2} \mathrm{eq} \mathrm{ano}{ }^{-1}$. Os valores calculados para as emissões de metano do projeto na lagoa secundária foram de $87,35 \mathrm{tCO}_{2} \mathrm{eq} \mathrm{ano}^{-1}$, e os valores de óxido nitroso emitidos na lagoa secundária foram de $4,31 \mathrm{tCO}_{2} \mathrm{eq} \mathrm{ano}^{-1}$. No total, o cenário do projeto resulta em $115,92 \mathrm{tCO}_{2} \mathrm{eq}$ ano $^{-1}$, conforme demonstrado na Tabela 1.

Por emissões antrópicas agregadas equivalentes de dióxido de carbono dos gases de efeito estufa, entende-se o somatório das emissões antrópicas de todos os gases de efeito estufa, convertidos para equivalente em $\mathrm{CO}_{2}$, utilizando-se do potencial de aquecimento global (PAG) de cada gás para 100 anos, conforme definido no IPCC-Painel Intergovernamental sobre Mudança do Clima (MIGUEZ, 2001).

Para o cenário da linha de base, a estimativa de emissões de metano foi de 436,77 $\mathrm{tCO}_{2}$ eq ano $^{-1}$, que são emitidos diretamente para a atmosfera, sendo a emissão total de óxido nitroso de $4,31 \mathrm{tCO}_{2} \mathrm{eq} \mathrm{ano}^{-1}$. No total, o valor das emissões estimadas da linha de base foi de $441,08 \mathrm{tCO}_{2} \mathrm{eq} \mathrm{ano}{ }^{-1}$, conforme descrito na Tabela 2.

TABELA 2. Emissões em $\mathrm{tCO}_{2} \mathrm{eq}$ ano $^{-1}$ detalhadas da linha de base por fontes.

\begin{tabular}{lc}
\hline Emissões da Linha de Base & $\mathrm{tCO}_{2} \mathrm{eq} \mathrm{ano}^{-1}$ \\
\hline $\mathrm{CH}_{4}$ da lagoa anaeróbia & 436,77 \\
$\mathrm{~N}_{2} \mathrm{O}$ da lagoa & 4,31 \\
Emissões totais do Projeto & 441,08 \\
\hline
\end{tabular}

Assim, o resultado do potencial estimativo de reduções é de $325,16 \mathrm{tCO}_{2} \mathrm{eq} \mathrm{ano}^{-1}$ (Tabela 3). Numa projeção para 10 anos, o total estimativo de reduções será de 3.251,60 tCO $\mathrm{tC}_{2}$ eq. HUBNER (2005), estudando uma unidade para 14.500 suínos, em sistema de ciclo completo, na região do extremo oeste do Paraná, estimou redução de $12.200 \mathrm{tCO}_{2} \mathrm{eq} \mathrm{ano}{ }^{-1}$.

TABELA 3. Reduções totais de emissões em $\mathrm{tCO}_{2}$ eq.

\begin{tabular}{lc}
\hline Reduções de Emissões & $\mathrm{tCO}_{2} \mathrm{eq} \mathrm{ano}^{-1}$ \\
\hline Reduções totais de emissões ano & 325,16 \\
Total -10 anos & $3.251,60$ \\
\hline
\end{tabular}

Na granja em estudo, de recria e de engorda de suínos, optou-se pela instalação do sistema de tratamento por meio de biodigestores. No entanto, em curto prazo, seria mais econômica a instalação de sistema de tratamento por lagoas anaeróbias. Segundo PERDOMO et al. (2003), o custo médio de implementação de um biodigestor é de US\$50,00 $\mathrm{m}^{-3}$ e de US\$5,00 $\mathrm{m}^{-3}$ para uma lagoa anaeróbia-padrão.

Pode-se, então, optar pela implantação do sistema de lagoas e não realizar investimentos dez vezes maiores, uma vez que eles estariam em conformidade com a legislação ambiental brasileira.

BELLI FILHO et al. (2001), realizaram estudo comparativo entre sistemas de tratamento por lagoas anaeróbias e biodigestores anaeróbios, acompanhando a evolução da demanda química de oxigênio e sólidos totais, cujos valores de redução desses parâmetros indicam que o reator UASB pode apresentar-se como alternativa às lagoas anaeróbias. O uso de biodigestores traz uma série de benefícios ambientais, tais como: redução do potencial poluidor (até $90 \%$ de matéria orgânica), formação de material estabilizado com baixa relação $\mathrm{C} / \mathrm{N}$ (10:1), aumento do teor de fósforo disponível, aumento na disponibilidade de macro e microelementos, menor perda de nitrogênio, melhoria nas condições físicas do solo, menor disseminação de plantas invasoras, ausência de cheiro desagradável, não atrai moscas e possibilita a aplicação diretamente sobre as culturas (PERDOMO et al., 2003). Outrossim, a possibilidade de redução de emissão de gases de efeito estufa e a obtenção de receitas por meio de créditos de carbono potencializam maior 
sustentabilidade da suinocultura, tanto no aspecto econômico, como no ambiental, principalmente considerando-se o médio ou longo prazo.

A utilização em larga escala de resíduos nitrogenados, resultando em importação de grande quantidade de nitrogênio para as unidades de produção intensiva de suínos, é identificada como um dos maiores problemas ambientais para a produção de animais nas áreas baixas na Europa. $\mathrm{O}$ desenvolvimento de sistemas que permitam o reúso desses resíduos gerados na produção de culturas locais, pode ser visto como solução sustentável para o problema (ATKINSON \& WATSON, 1996).

De acordo com ABBOZZO et al. (1996), a produção de suínos será sustentável se manter ou melhorar a qualidade do ambiente e dos recursos naturais, a qualidade de vida dos produtores e da sociedade, os benefícios aos produtores e a qualidade da carne produzida.

Para o sucesso a longo prazo, as dimensões ambiental, social e econômica da produção de suínos necessitam ser cuidadosamente consideradas nas granjas, comunidades rurais, consumidores e no ecossistema (HONEYMAN, 1996).

No caso específico do presente trabalho, o valor anual estimado de Redução de Emissão Certificada (CER) é de 325,16 $\mathrm{tCO}_{2} \mathrm{eq}$, o que possibilitaria redução de emissão de $0,54 \mathrm{tCO}_{2} \mathrm{eq} \mathrm{ano}^{-1}$ animal $^{-1}$. HUBNER (2005) encontrou valor de $0,84 \mathrm{tCO}_{2} \mathrm{eq}$ ano ${ }^{-1}$, para granja independente de 14.500 animais em ciclo completo. Já TAMMINGA \& VERSTEGEN (1992) citam que um suíno de $50 \mathrm{~kg}$ pode produzir cerca de $450 \mathrm{~kg} \mathrm{CO}_{2} \mathrm{eq} \mathrm{ano}{ }^{-1}$.

CENAMO (2005) indica que, ainda sujeitos às grandes variações entre os acordos bilaterais negociados, os preços atuais das potenciais CERs (expressas em $\mathrm{tCO}_{2} \mathrm{eq}$ ) têm estado em torno de quatro a seis Euros $(€)$. O preço atualmente é de US\$ 5,63 tCO $\mathrm{eq}^{-1}$ (BRASIL, 2005).

Já HUBNER (2005) indica que as ofertas pelos créditos giram em torno de US\$ 4,00 $\mathrm{tCO}_{2} \mathrm{eq}^{-1}$. Procedendo-se a uma análise, a partir de um valor mais conservador, a granja em estudo poderá obter acréscimo de receita anual de $\mathrm{R} \$ 3.186,00$ (três mil, cento e oitenta e seis reais), sendo, em valores financeiros, $\mathrm{R} \$ 5,31 \mathrm{ano}^{-1} \mathrm{animal}^{-1}$.

O setor agropecuário tem enorme potencial de contribuição no controle das mudanças climáticas. Hoje ele é globalmente responsável por $20 \%$ da emissão de gases do efeito estufa. O Brasil tem a quarta suinocultura do mundo em população animal, e também é um dos primeiros em qualidade e eficiência de produção. Essa importante indústria está sempre sintonizada no que há de mais atual em tecnologias nos diferentes setores.

O destino adequado dos dejetos em sistemas intensivos de criação é tema de discussões há décadas. Por muito tempo, foi considerado como problema e, de forma geral, é indicativo prever que o Protocolo de Kioto pode contribuir com o papel de mudar o paradigma da busca pela sustentabilidade da suinocultura, tanto no aspecto ambiental, como no social e econômico.

Não foi levado em conta o custo para elaboração do Documento de Concepção (PDD) do projeto, que poderia ser elaborado de forma conjunta com outros produtores; no entanto, atualmente, esse custo é um limitante para efetivação desse processo.

\section{CONCLUSÕES}

Pôde-se inferir, por meio da estimativa deste estudo, que o sistema de manejo de dejetos de suínos utilizando biodigestores é um eficiente instrumento de redução de emissão de $\mathrm{CO}_{2}$ eq, desde que o metano seja queimado ou utilizado para outros fins, como, por exemplo, a geração de energia.

Para a granja em sistema de terminação com 600 animais alojados, o potencial de redução de carbono foi de $325,16 \mathrm{tCO}_{2} \mathrm{eq}^{1} \mathrm{ano}^{-1}$. 
Com a comercialização da Redução Certificada de Emissões, a granja poderá obter acréscimo de receita anual de $\mathrm{R} \$ 3.186,00$ (três mil, cento e oitenta e seis reais), ou $\mathrm{R} \$ 5,31$ animal $^{-1}$ ano $^{-1}$. Considerando as dificuldades financeiras por que a atividade vem passando e a necessidade de adequação ambiental das propriedades, o valor acima contribui de forma significativa no aumento das receitas da atividade.

\section{REFERÊNCIAS}

ABBOZZO, P.; BOGGIA, A.; BRUNETTI, M. Environmental quality and hog production. Environmental Monitoring and Assessment, Netherlands, v.41, n.3, p.171-82, 1996.

ATKINSON, D.; WATSON, C.A. The environmental impact of intensive systems of animal production in the lowlands. Animal Science, Penicuik, v.63, n.5, p.353-61, 1996.

BELLI FILHO, P.; CASTILHOS JÚNIOR, A.B.; COSTA, R.H.R.; SOARES, S.R.; PER DOMO,C.C. Tecnologias para o tratamento de dejetos de suínos. Revista Brasileira de Engenharia Agrícola e Ambiental, Campina Grande, v.5, n.1, p.166-70, 2001.

BENEDI, J.M.H. El ambiente de los alojamientos ganaderos. Madrid: Ministerio de la Agricultura, Pesca y Alimentación, Servicio de Extensión Agraria, 1986. 28 p. (Hojas Divulgadoras, 6).

BRASIL. Ministério da Agricultura, Pecuária e Abastecimento. Plano nacional de agroenergia 2006 - 2011. Brasília: EMBRAPA, 2005. 120 p.

CENAMO, M.C. O Mercado de carbono e as oportunidades para o agronegócio brasileiro. Piracicaba: CEPEA/DEAS/ESALQ/USP, Set. 2005. 4 p. Disponível em: http://www.cepea.esalq.usp.br/pdf/beefpoint_cepea.pdf. Acesso em: 31 out. 2005.

ERNST, M.; RODECKER, J.; LUVAGA, E.; ALEXANDER, T.; KLIEBENSTEIN, J.; MIRANOWSKI, J. Viability of methane production by anaerobic digestion on Iowa swine farms. Ames, Iowa State University, Departament of Economics, 1990. 7 p.

HONEYMAN, M.S. Sustainability issues of U.S. swine production. Journal of Animal Sciences, Ames, v.74, n.1, p.1410-17, 1996.

HUBNER, T. Análise da viabilidade da reciclagem e aproveitamento de dejetos de suínos. 2005. 77 f. Monografia (Especialização em Agronegócio) - Escola Superior de Agricultura "Luiz de Queiroz", Universidade de São Paulo, Piracicaba, 2005.

IAPAR. INSTITUTO AGRONÔMICO DO PARANÁ. Cartas climáticas básicas do Estado do Paraná. Curitiba, 1994. 49 p.

IBGE. INSTITUTO BRASILEIRO DE GEOGRAFIA E ESTATÍSTICA. Mapas de climas do Brasil. Disponível em: http://www.mapas.ibge.gov.br/website/clima/viewer.htm. Acesso em: 25 de out. 2005.

IPCC. INTERGOVERNAMENTAL PANEL ON CLIMATE CHANGE. Climate Change 1994. Radiative Forcing of Climate Change. Cambridge: University Press, 1995. "pag. irreg."

IPCC. INTERGOVERNAMENTAL PANEL ON CLIMATE CHANGE. Directrices del IPCC para los inventários nacionales de gases de efecto invernadero, versión revisada em 1996. Reino Unido: IPCC WGI Technical Support Unit, 1997.

KIRCHMANN, H.; ESALA, M.; MORKEN, J.; FERM, M.; BUSSINK, W.; GUSTAVSSON, J.; JAKOBSSON, C. Ammonia emissions from agriculture. Nutrient Cycling in Agroecosystems, Bonn, v.51, n.1, p.1-3, 1998.

LIMA, A.L.; PESSOA, M.C.P.Y.; LIGO, M.A.V. Primeiro inventário brasileiro de emissões antrópicas de gases de efeito estufa: Relatórios de referência - Emissões de metano da pecuária. Brasília: IBGE-EMBRAPA - MCT, 2002. 79 p. 
LIMA, M.A.; BOEIRA, R.C.; CASTRO, V.L.S.S.; LIGO, M.A.V.; CABRAL, O.M.R. Estimativas das emissões de gases de efeito estufa proveniente de atividades agrícolas no Brasil. In: LIMA, M.A.; CABRAL, O.M.R.; MIGUEZ, J.D.G. (Ed.) Mudanças climáticas globais e a agropecuária brasileira. Jaguariúna: EMBRAPA/CNPMA, 2001. cap. 7, p.169-89.

LUCAS JÚNIOR, J. Algumas considerações sobre o uso do estrume de suínos como substrato para três sistemas de biodigestores anaeróbios. 1994. 113f. Tese (Livre-Docência) - Faculdade de Ciências Agrárias e Veterinárias, Universidade Estadual Paulista, Jaboticabal, 1994.

MIGUEZ, J.D.G. A Convenção-Quadro das Nações Unidas sobre Mudança do Clima e o Protocolo de Quioto. In: LIMA, M.A.; CABRAL, O.M.R.; MIGUEZ, J.D.G. (Ed.) Mudanças climáticas globais e a agropecuária brasileira. Jaguariúna: EMBRAPA/CNPMA, 2001. cap. 1, p. 17-30.

OENEMA, O.; WRAGE, N.; VELTHOF, G.L.; GROENIGEN, J.W.V.; DOLFING, J.;

KUIKMAN, P.J. Trends in global nitrous oxide emissions from animal production systems.

Nutrient Cycling in Agroecosystems, Bonn, v.72, n.1, p.51-65, may, 2005.

OLIVEIRA, P.A.V. Produção e aproveitamento do biogás. In: OLIVEIRA, P.A.V. (Coord.)

Tecnologias para o manejo de resíduos na produção de suínos: manual de boas práticas.

Concórdia: EMBRAPA-CNPSA, 2004. cap. 4, p.43-55.

OLIVEIRA, P.A.V.; HIGARASHI, M.M.; NUNES, M.L.A. Emissões de gases, na suinocultura, que provocam o Efeito Estufa. Concórdia: EMBRAPA - CNPSA, 2004. 12 p.

PERDOMO, C.C.; LIMA, G.J.M.M.; NONES, K. Suinocultura e estratégias para a redução de gases e poluentes. In: LIMA, M.A.; CABRAL, O.M.R.; MIGUEZ, J.D.G. (Ed.) Mudanças climáticas globais e a agropecuária brasileira. Jaguariúna: EMBRAPA/CNPMA, 2001. cap. 16, p.325-45.

PERDOMO, C.C; OLIVEIRA, P.A.; KUNZ, A. Sistemas de tratamento de dejetos de suínos: inventário tecnológico. Concórdia: EMBRAPA-CNPSA, 2003. 83 p. (Documentos, 85).

TAMMINGA, S.; VERSTEGEN, N.W.A. Implication of nutririon of animals on environmenral pollution: In: GARNSWORTH, P.C.; HARGSIGN, W.; COLE, D.I.A. (Ed.) Recent advances in nutrition. Oxford: Butterworth-Heinemann, 1992. p.113-130.

UFCCC. UNITED NATIONS FRAMEWORK CONVENTION ON CLIMATE CHANGE. GHG emission reductions from manure management systems. 2004. Disponível em: http://cdm.unfccc.int/methodologies/PAmethodologies/approved.html. Acesso em: 29 out. 2005.

USEPA. UNITED STATES ENVIRONMENTAL PROTECTION AGENCY. International anthropogenic methane emissions: estimates for 1990. Washington: Office of Policy Planning and Evaluation, 1994. $160 \mathrm{p}$. 